\title{
ON THE LIMIT DISTRIBUTIONS OF $k$ TH ORDER STATISTICS FOR SEMI-PARETO PROCESSES
}

Abstract. Asymptotic properties of the $k$ th largest values for semi-Pareto processes are investigated. Conditions for convergence in distribution of the $k$ th largest values are given. The obtained limit laws are represented in terms of a compound Poisson distribution.

1. Introduction. Pillai [5] has discussed semi-Pareto processes, of which Pareto processes form a proper sub-class. He has examined asymptotic properties of the maximum and minimum of the first $n$ observations. We here obtain conditions for convergence in distribution of the $k$ th largest values for semi-Pareto processes.

We say that a random variable $X$ has semi-Pareto distribution and write $X \sim P_{S}(\alpha, p)$ if its survival function is of the form

$$
\bar{F}_{X}(x)=1-F_{X}(x)=P(X>x)=\frac{1}{1+\psi(x)}, \quad x \geq 0,
$$

where $\psi(x)$ satisfies the functional equation

$$
\psi(x)=\frac{1}{p} \psi\left(p^{1 / \alpha} x\right),
$$

where $\alpha>0$ and $0<p<1$.

The autoregressive semi-Pareto model ARSP(1) is built using a sequence of independent identically distributed (i.i.d.) random variables in the following manner ([5]). Let $\left\{\varepsilon_{n}, n \geq 1\right\}$ be i.i.d. $P_{S}(\alpha, p)$ random variables and

1991 Mathematics Subject Classification: Primary 60G70; Secondary 69J05.

Key words and phrases: extreme values, autoregressive process, semi-Pareto process. 
for each $n=1,2, \ldots$ define

(2) $\quad X_{n}= \begin{cases}p^{-1 / \alpha} X_{n-1} & \text { with probability } p, \\ \min \left(p^{-1 / \alpha} X_{n-1}, \varepsilon_{n}\right) & \text { with probability } 1-p\end{cases}$

The process defined by (2) will be called an $\operatorname{ARSP}(1)$ process.

The ARSP(1) process is clearly Markovian. If the initial distribution is $X_{0} \sim P_{S}(\alpha, p)$, then $X_{n} \sim P_{S}(\alpha, p)$ and the process is strictly stationary.

In particular, if $\left\{\varepsilon_{n}, n \geq 1\right\}$ is a sequence of i.i.d. random variables with common distribution of the Pareto form

$$
P\left(\varepsilon_{1}>x\right)=\left[1+(x / \sigma)^{1 / \gamma}\right]^{-1}, \quad x \geq 0,
$$

where $\sigma>0$ and $\gamma>0$, we obtain the autoregressive Pareto $(\mathrm{ARP}(1))$ process $([7])$.

2. Level crossing processes. Let $\left\{X_{n}, n \geq 1\right\}$ be an $\operatorname{ARSP}(1)$ process. For each $n \geq 1$, let $M_{n}^{(1)} \geq M_{n}^{(2)} \geq \ldots \geq M_{n}^{(n)}$ be the order statistics of $X_{1}, \ldots, X_{n}$. The problem is to study the limiting behaviour of the $k$ th order statistics $M_{n}^{(k)}$ for any fixed $k \geq 1$ as $n \rightarrow \infty$. The asymptotic distribution of $M_{n}^{(k)}$ will be obtained by considering the number of exceedances of a level $x$ by $X_{1}, \ldots, X_{n}$.

For any $x>0$, we define the level crossing process $Z_{n}(x)$ associated with $\left\{X_{n}\right\}$ by

$$
Z_{n}(x)= \begin{cases}1 & \text { if } X_{n}>x \\ 0 & \text { if } X_{n} \leq x\end{cases}
$$

(cf. $[1,5,7]$ ). The two-state stochastic process $\left\{Z_{n}(x), n \geq 1\right\}$ turns out to be a Markov chain with transition matrix

$$
P=\frac{1}{1+\psi(x)}\left[\begin{array}{cc}
p+\psi(x) & 1-p \\
(1-p) \psi(x) & 1+p \psi(x)
\end{array}\right]
$$

The obvious relation

$$
P\left(M_{n}^{(k)} \leq x\right)=P\left(\sum_{j=1}^{n} Z_{j}(x)<k\right), \quad-\infty<x<\infty,
$$

will play a role in this paper.

3. Asymptotic distributions of $k$ th order statistics. Suppose that, for $\tau>0$, there exists a sequence $\left\{u_{n}=u_{n}(\tau)\right\}$ such that

$$
\lim _{n \rightarrow \infty} n \bar{F}_{X}\left(u_{n}(\tau)\right)=\tau
$$

where $\bar{F}_{X}$ is given by (1). 
We shall investigate properties of the random variable

$$
S_{n}(\tau)=\sum_{j=1}^{n} Z_{j}\left(u_{n}(\tau)\right)
$$

for some fixed $\tau>0$, as $n \rightarrow \infty$, and as consequences, we shall obtain limiting distributional results for the $k$ th order statistics. The main tool is a result which gives conditions for the convergence in distribution of sums of 0-1 Markov chains to a compound Poisson distribution (cf. $[2,4,6])$.

TheOREM 1. Let $\left\{Y_{n, j}, j=1, \ldots, n\right\}, n=1,2, \ldots$, be a sequence of two-state 0 and 1 homogeneous Markov chains, with transition matrices

$$
\left[\begin{array}{cc}
1-(1-\pi) \varrho_{n} & (1-\pi) \varrho_{n} \\
(1-\pi)\left(1-\varrho_{n}\right) & (1-\pi) \varrho_{n}+\pi
\end{array}\right]
$$

where $0 \leq \varrho_{n} \leq 1$ and $0 \leq \pi \leq 1$, and initial probabilities

$$
P\left(Y_{n, 1}=1\right)=1-P\left(Y_{n, 1}=0\right)=\varrho_{n} .
$$

If

then for $k=0,1,2, \ldots$,

$$
\lim _{n \rightarrow \infty} n \varrho_{n}=\lambda, \quad \lambda>0,
$$

$$
\lim _{n \rightarrow \infty} P\left(\sum_{j=1}^{n} Y_{n, j}=k\right)=T(k,(1-\pi) \lambda, 1-\pi),
$$

where

(8) $T(k, \lambda, r)= \begin{cases}e^{-\lambda} & \text { for } k=0, \\ \sum_{m=1}^{k} C_{k-1}^{m-1}(1-r)^{k-m} r^{m} \frac{\lambda^{m}}{m !} e^{-\lambda} & \text { for } k=1,2, \ldots\end{cases}$

Therefore, the limit law for $\sum_{j=1}^{n} Y_{n, j}$ is of the compound Poisson type.

Consider now, for some $\tau>0$, the sequence of Markov chains

$$
Y_{n, j}=Z_{j}\left(u_{n}(\tau)\right), \quad j=1, \ldots, n .
$$

The transition matrices for the sequence (9) are of the form (7) with

$$
\pi=p, \quad \varrho_{n}=\frac{1}{1+\psi\left(u_{n}(\tau)\right)} .
$$

Note that, by the condition (6), we have

$$
\lim _{n \rightarrow \infty} n \varrho_{n}=\lim _{n \rightarrow \infty} n \bar{F}_{X}\left(u_{n}(\tau)\right)=\tau>0 .
$$

Finally, it follows from Theorem 1 that

$$
\lim _{n \rightarrow \infty} P\left(S_{n}(\tau)=k\right)=T(k,(1-p) \tau, 1-p), \quad k=0,1,2, \ldots
$$


We shall use the results (11) to study the limit laws for the $k$ th order statistics of semi-Pareto processes.

THEOREM 2. Let $\left\{X_{n}, n \geq 1\right\}$ be a strictly stationary $A R S P(1)$ process. Suppose that, for $\tau>0$, there exists a sequence $\left\{u_{n}(\tau), n \geq 1\right\}$ such that

$$
\lim _{n \rightarrow \infty} \frac{1}{n} \psi\left(u_{n}(\tau)\right)=\frac{1}{\tau},
$$

where $\psi$ is given by (1). Then, for each $k=0,1,2, \ldots$,

$$
\lim _{n \rightarrow \infty} P\left(M_{n}^{(k)} \leq u_{n}(\tau)\right)=\sum_{j=0}^{k-1} T(j,(1-p) \tau, 1-p),
$$

where the function $T(k, \lambda, r)$ is defined by (8).

Proof. From (5) we have

$$
P\left(M_{n}^{(k)} \leq u_{n}(\tau)\right)=P\left(\sum_{j=1}^{n} Z_{j}\left(u_{n}(\tau)\right)<k\right), \quad k=1, \ldots, n,
$$

where $Z_{j}(x)$ are defined by (4). Thus, by (10)-(12), we obtain the desired result.

The case $k=1$ of Theorem 2 shows that

$$
\lim _{n \rightarrow \infty} P\left(M_{n}^{(1)} \leq u_{n}(\tau)\right)=\exp (-(1-p) \tau)
$$

In particular, if $\left\{X_{n}, n \geq 1\right\}$ is a strictly stationary Pareto process with $\bar{F}_{X_{n}}$ given by (3), then we have $\psi(x)=(x / \sigma)^{1 / \gamma}$, and hence (12) holds with $u_{n}(\tau)=\sigma n^{\gamma} x, \tau=x^{-1 / \gamma}, x>0$. Thus, from (14) we obtain the result which is due to Yeh et al. ([7], Equation (3.8)):

$$
P\left(M_{n}^{(1)} \leq \sigma n^{\gamma} x\right)= \begin{cases}\exp \left(-(1-p) x^{-1 / \gamma}\right) & \text { if } x>0 \\ 0 & \text { if } x \leq 0\end{cases}
$$

\section{References}

[1] B. C. Arnold and J. T. Hallett, A characterization of the Pareto process among stationary stochastic processes of the form $X_{n}=c \min \left(X_{n-1}, Y_{n}\right)$, Statist. Probab. Lett. 8 (1989), 377-380.

[2] J. Gani, On the probability generating function of the sum of Markov Bernoulli random variables, J. Appl. Probab. 19A (1982), 321-326.

[3] M. R. Leadbetter, G. Lindgren and H. Rootzén, Extremes and Related Properties of Random Sequences and Processes, Springer, New York, 1983.

[4] J. Pawłowski, Poisson theorem for non-homogeneous Markov chains, J. Appl. Probab. 26 (1989), 637-642.

[5] R. N. Pillai, Semi-Pareto processes, ibid. 28 (1991), 461-465.

[6] Y. H. Wang, On the limit of the Markov binomial distribution, ibid. 18 (1981), 937-942. 
[7] H. C. Yeh, B. C. Arnold and C. A. Robertson, Pareto processes, ibid. 25 (1988), 291-301.

Magdalena Chrapek

Institute of Mathematics

Pedagogical University

ul. M. Konopnickiej 21

25-406 Kielce, Poland

Wiesław Dziubdziela

Faculty of Civil and Environment Engineering

Department of Building Constructions

Czȩstochowa Technical University

ul. Akademicka 3

42-200 Czȩstochowa, Poland
Jadwiga Dudkiewicz

Department of Mathematics Kielce University of Technology

Al. Tysiąclecia Państwa Polskiego 7 25-314 Kielce, Poland

Received on 30.10.1995;

revised version on 18.1.1996 